\title{
Development and metrological characterization of an aerosol generation device dedicated to inhalation toxicology studies: the nanopesticide case
}

\author{
Quentin Hamdaoui ${ }^{1}$, François Gaie-Levrel ${ }^{1}$, Tatiana Macé $^{1}$, Sophie Vaslin-Reimann ${ }^{1}$, Frédéric Flamant ${ }^{2}$, and Anna \\ Bencsik $^{3}$ \\ ${ }^{1}$ Laboratoire National de métrologie et d'Essais (LNE), 1 rue Gaston Boissier, 75724 Paris Cedex 15, France \\ ${ }^{2}$ Institut de Génomique Fonctionnelle de Lyon (IGFL), 32-34 Avenue Tony Garnier, 69007 Lyon Cedex 07, France \\ ${ }^{3}$ Université Lyon 1 - Agence nationale de sécurité sanitaire de l'alimentation, de l'environnement et du travail (ANSES), 31 avenue Tony \\ Garnier, 69364 Lyon, France
}

\begin{abstract}
Despite the controversies surrounding the potential health effects associated with engineered nanomaterials, novel agrochemicals combining nanotechnology and pesticides are emerging. These products, named nanopesticides, are being developed to improve the efficiency of conventional agrochemicals. However, they represent an intentional anthropogenic source of nanomaterials within the different environmental compartments which constitutes a possible exposure of agricultural populations notably via the aerosols generated by farming activities. The hazard related to this new type of contaminants must be assessed by using inhalation toxicology studies that are designed to reproduce the complexity of these aerosols exposure, in order to be relevant for human health studies. In the present article, we report an experimental strategy combining both the recommendations in animal experimentation and the OECD guidelines for chemicals testing. To explore the neurotoxicity linked with the chronic exposure to aerosols generated from a nanopesticide, we develop an original device dedicated to inhalation toxicology with rodents. Through this proof of concept study, our cross-disciplinary project aims at proposing a validated methodology to study the inhalation toxicity of complex formulations represented by nanopesticides.
\end{abstract}

\section{Introduction}

In response to the worldwide growing demand for food, nanotechnology applications in agriculture elicit a particular interest. A wide range of novel products called nanopesticides (NPEs) are emerging on the market [1] thanks to engineered nanomaterials (ENMs) used either as active ingredients or additives [2]. Due to unique properties granted by their nanometric scale, these new products would theoretically benefit from enhanced efficiency compared to conventional pesticides while mitigating the principal drawbacks associated with the exposure of non-target organisms. They aim at responding to the weaknesses of current plant protection products mostly by alleviating the environmental footprint of modern agriculture which is one of the main sources of ecosystem contaminations. As the fate of NPEs is still poorly understood, they have been integrated as a priority by various regulatory bodies and research funding agencies such as the USDA [3]. The use of NPEs could result ultimately in intentional and diffuse inputs of ENMs into the environment, which is a topic of great concern. Inhalation is often considered as the most likely exposure pathway for professionals handling aerosolizable ENMs. Accordingly, agricultural populations may be specifically exposed through inhalation especially because of the spray drift hazard.
However, studies on the potential impact of chronic exposure to NPEs via inhalation are almost inexistent and they mainly rely on in vivo assays. Moreover, such studies are rarely in accordance with the OECD guidelines for the testing of chemicals [4].

The aerosol exposure device presented in this paper is dedicated to whole-body inhalation studies with rodents. Considering the important need for harmonizing the procedures for aerosols characterization, specific cares were taken to make a compromise between OECD guidelines for chemical testing [4] and the necessary conditions to be hosted in any animal facility. It has been designed to conform to standard features: (1) to comply with ethics and hosting parameters in accordance with animal welfare; (2) to enable an estimation of the dosimetry by generating stable aerosols for two hours; (3) to optimize the repeatability and the reproducibility of the measured parameters in accordance with the ISO 5725-2 standard [5]; (4) to deliver an homogeneous test atmosphere in the exposure chamber; (5) to protect operators from ENM. The whole-body exposure appears to be the most realistic and accurate way to expose rodents chronically to an aerosol by enabling animals to behave naturally during the exposure phases. In order to manage the upcoming toxicology study, this ad hoc device has been characterized with a toxicological model 
of NPE composed of nano- $\mathrm{TiO}_{2} \mathrm{P} 25$ and paraquat (PQ) which is the active ingredient of a widely used herbicide.

According to the actual data on the production and the life cycle of the main ENMs in European Union, the environmental contamination by nano- $\mathrm{TiO}_{2}$ is expected to be important [6]. Nano- $\mathrm{TiO}_{2}$ has been chosen as a model compound because it could be included in NPEs as an active substance [7] and/or as an additive within a pesticide formulation. The relevance of this model relies essentially in the neurotoxicity of $\mathrm{PQ}$, which is known to induce a parkinsonian syndrome both in humans and animals [8]. Indeed, several epidemiological studies associate the professional exposure to paraquat, with the incidence of Parkinson disease among farmers [9]. We plan to use nano- $\mathrm{TiO}_{2}$ and $\mathrm{PQ}$ in combination, to investigate the potential synergistic toxic effect that could occur on the nervous system of chronically exposed rodents.

The experimental design will be built in order to assess the neurotoxicity of chronic inhalation of the compounds of interest both during gestation and adulthood. Mice will be exposed 2h/day, 5 days/week, during gestation or for 8 weeks to relevant doses of nano- $\mathrm{TiO}_{2}$ and $\mathrm{PQ}$ independently and in combination (nano- $\mathrm{TiO}_{2}+\mathrm{PQ}$ ). To this end, two mouse lines will be used: a wild type (C57B1/6) and a transgenic line (TgM83), the latest being predisposed to the development of a neurodegenerative disease [10]. Neurotoxicity will be assessed using a combination of parameters such as measurements of locomotor ability, histopathology, inflammation, oxidative stress and transcriptomic analyses.

\section{Methods}

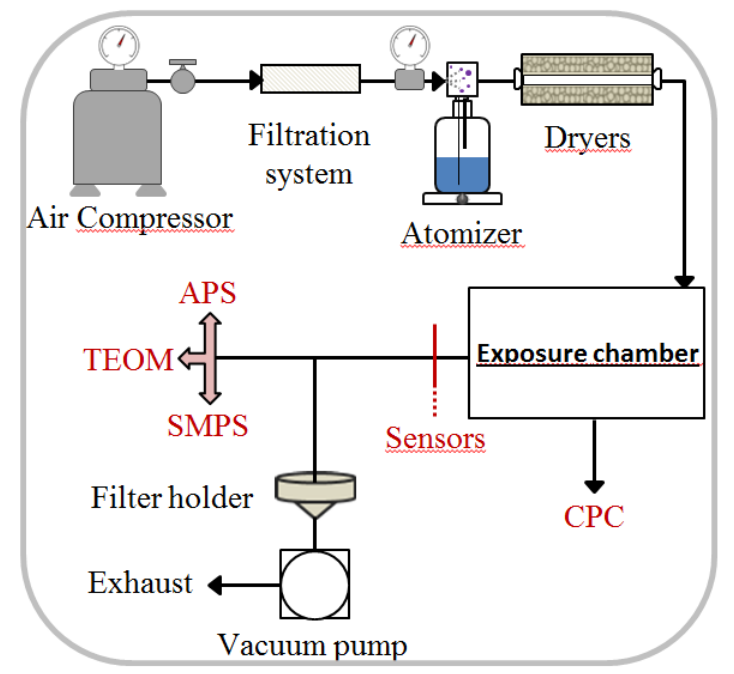

Fig. 1. Scheme of the experimental device

Our exposure device is built by coupling a whole-body chamber with an aerosol nebuliser (Figure 1). The chamber is a rodent cage made out of polysulfone plastic used in animal facility which is sealed and customized to inject and sample aerosols. Moreover, a valve enables to monitor the particles concentrations within the exposure chamber using a condensation particle counter (CPC 3007, TSI). This real-time monitoring ensures the metrological characterization and it guarantees the proper functioning of the device during the animal exposure.

The aerosol is generated from a colloidal suspension of nano- $\mathrm{TiO}_{2} \mathrm{P} 25$ and $\mathrm{PQ}$ powders in ultrapure water. Thanks to a collision jet nebulizer (TSI 3076), this system produces droplets using pressurized air (2.5 bars) coming from a silent compressor $(42 \mathrm{~dB})$ to avoid animal stress due to noise. Then, the aerosols pass through diffusions dryers which are regenerated before use to obtain a steady hygrometry for two hours. A clean air flow, regulated by mass flow controllers, is chosen to be adequate for both the aerosol homogenization inside the chamber and the animal welfare. A multiinstrumental set-up composed of a real-time monitoring and off-line aerosols samplings is used to characterize mass and number concentrations, particle number size distributions, particle morphology (electron microscopy) and environmental parameters of animals (hygrometry, temperature, pressure). To prevent any leak and ensure the operator safety, the chamber is placed under a slight negative internal pressure thanks to the pumping at the outlet of the chamber. The maximal operating noise and the air renewal are determined to match the best as possible the recommended conditions in animal facilities.

\section{Results and discussion}

Table 1. Results of the metrological characterization

\begin{tabular}{lccc} 
& $\mathbf{P Q}$ & $\mathrm{TiO}_{2}$ & $\mathbf{P Q}+\mathrm{TiO}_{2}$ \\
\hline \multirow{2}{*}{$\mathbf{C}_{\text {mass }}\left(\mathrm{mg} / \mathrm{m}^{3}\right)$} & $10,1 \times 10^{-2}$ & 10.6 & 10.7 \\
& $3.2 \%$ & $8.6 \%$ & $4.1 \%$ \\
\hline $\mathbf{C}_{\text {number }}$ & $2.5 \times 10^{5}$ & $2.0 \times 10^{5}$ & $2.5 \times 10^{5}$ \\
$\left(\right.$ part $\left./ \mathrm{cm}^{3}\right)$ & $2.4 \%$ & $5.1 \%$ & $7.8 \%$ \\
\hline \multirow{2}{*}{$\mathbf{d}_{\text {median }}(\mathrm{nm})$} & 50.0 & 220.3 & 173.3 \\
\hline \hline
\end{tabular}

Table 1 summarizes the main results of the characterization measurements conducted in the exposure chamber. Ten assays of two hours were performed for each aerosol (PQ, nano- $\mathrm{TiO}_{2}$ and $\mathrm{PQ}+$ nano- $\mathrm{TiO}_{2}$ ). The results are expressed as mass concentration $\left(\mathrm{C}_{\text {mass }}\right.$ in $\left.\mathrm{mg} / \mathrm{m}^{3}\right)$, number concentration $\left(\mathrm{C}_{\text {number }}\right.$ in particles $\left./ \mathrm{cm}^{3}\right)$, and median statistic diameter $\left(\mathrm{d}_{\text {median }}\right.$ in $\left.\mathrm{nm}\right)$ with their corresponding relative reproducibility standard deviation calculated according to the ISO 5725-2 standard [5] (in brackets). According to OECD recommendation for aerosols assessment, the concentrations measured by individual samplings should not differ from the mean concentration by more than $20 \%$. The results are therefore in accordance with such recommendations and the test atmosphere can be considered as steady. The chamber temperature $(24.6 \pm$ $\left.0.9^{\circ} \mathrm{C}\right)$, the hygrometry $(19.6 \pm 1.2 \%)$ and the chamber internal pressure $(-0.60 \pm 0.04$ mbar $)$ were monitored thanks to adequate calibrated sensors. The results of the measurements of the mean mass concentrations are presented in Figure 1. 


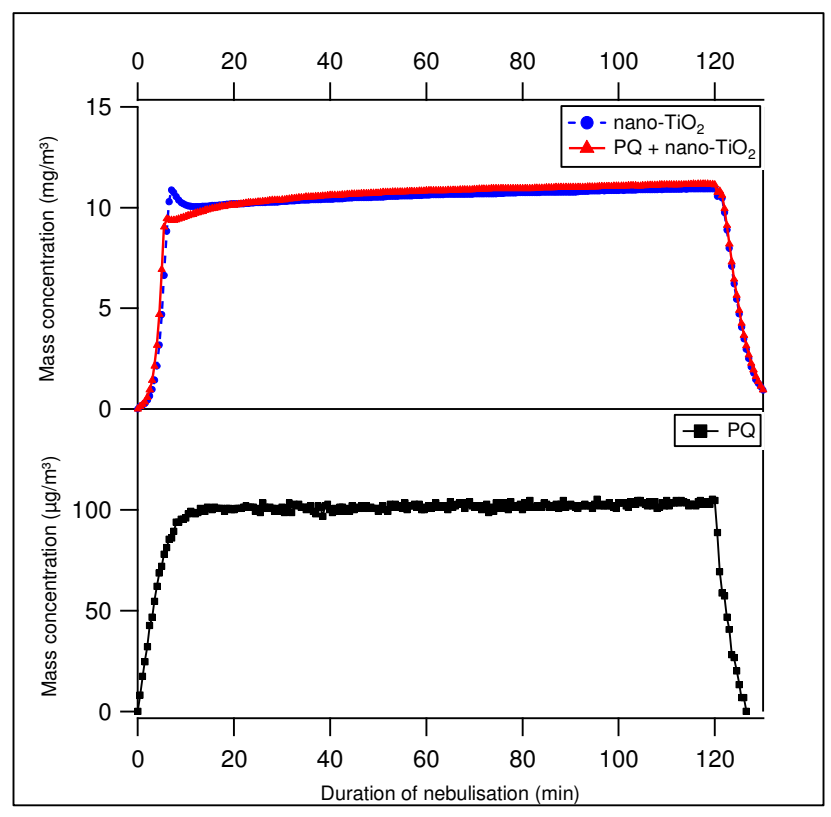

Fig. 2. Real-time total mass concentrations measurements for $120 \mathrm{~min}$ of experimentation.

\section{Conclusions}

NPEs appear as an opportunity for modern agriculture to respond to the worldwide growing demand for food. But the safety of these new products still raises many questions especially concerning the potential health effects of such mixtures. In this context, validated methodologies to study the inhalation toxicity of complex formulations are necessary. Accordingly, we developed and characterized a dedicated device which enables the upcoming NPE neurotoxicity study conducted with a murine model. Finally, it will participate to the production of relevant toxicological data which are required for the health risk assessment related to the chronic exposure to aerosols containing ENMs and pesticides.

This work is carried out as part of a thesis supported by a CIFRE fellowship involving the collaboration of the LNE and the École Normale Supérieure de Lyon (ENS Lyon).

\section{References}

1. S. Kumar, M. Nehra, N. Dilbaghi, G. Marrazza, A. A. Hassan, and K.-H. Kim, J. Controlled Release 294, 131 (2019)

2. M. Kah and T. Hofmann, Environ. Int. 63, 224 (2014)

3. USDA (2015). https://nifa.usda.gov/pressrelease/usda-awards-38-million-grantsnanotechnology-research [Accessed 05/06, 2019].
4. OCDE (2018), Test No. 413: Subchronic Inhalation Toxicity: 90-day Study, OECD Guidelines for the Testing of Chemicals

5. ISO 5725-2 (1994) Accuracy of measurement methods and results - part 2: basic method for the determination of repeatability and reproducibility of a standard measurement method

6. T. Y. Sun, F. Gottschalk, K. Hungerbühler, and B. Nowack, Environ. Pollut. 185, 69 (2014)

7. M. F. La Russa, A. Macchia, S. A. Ruffolo, F. De Leo, M. Barberio, P. Barone, G. M. Crisci, and C. Urzì, Int. Biodeterior. Biodegrad. 96, 87 (2014)

8. S. Bastías-Candia, J. M. Zolezzi, and N. C. Inestrosa, Mol. Neurobiol. 56, 1044 (2019)

9. C. M. Tanner, F. Kame, G. W. Ross, J. A. Hoppin, S. M. Goldman, M. Korell, C. Marras, G. S. Bhudhikanok, M. Kasten, A. R. Chade, K. Comyns, M. B. Richards, C. Meng, B. Priestley, H. H. Fernandez, F. Cambi, D. M. Umbach, A. Blair, D. P. Sandler, and J. W. Langston, Environ. Health Perspect. 119, 866 (2011)

10. B. I. Giasson, J. E. Duda, S. M. Quinn, B. Zhang, J. Q. Trojanowski, and V. M.-Y. Lee, Neuron 34, 521 (2002) 